\title{
Linx
}

Revue des linguistes de l'université Paris X Nanterre

$42 \mid 2000$

Approches sociolinguistiques du plan phonique

\section{Robert FOREST (1999), Empathie et linguistique}

Paris, Presses universitaires de France, coll. Linguistique nouvelle, 238 p.

Philippe Bourdin

\section{OpenEdition}

Journals

Édition électronique

URL : http://journals.openedition.org/linx/994

DOI : $10.4000 / \operatorname{lin} x .994$

ISSN : 2118-9692

Éditeur

Presses universitaires de Paris Nanterre

Édition imprimée

Date de publication : 1 juin 2000

Pagination : 182-188

ISSN : 0246-8743

Référence électronique

Philippe Bourdin, «Robert FOREST (1999), Empathie et linguistique », Linx [En ligne], 42 | 2000, mis en ligne le 05 juillet 2012, consulté le 22 septembre 2020. URL : http://journals.openedition.org/linx/994 ; DOI : https://doi.org/10.4000/linx.994

Ce document a été généré automatiquement le 22 septembre 2020

Département de Sciences du langage, Université Paris Ouest 


\title{
Robert FOREST (1999), Empathie et linguistique
}

Paris, Presses universitaires de France, coll. Linguistique nouvelle, 238 p.

\author{
Philippe Bourdin
}

\section{RÉFÉRENCE}

Robert FOREST (1999), Empathie et linguistique, Paris, Presses universitaires de France, coll. Linguistique nouvelle, $238 \mathrm{p}$.

\section{De l'empathie, comme principe explicatif en morpho- syntaxe}

1 Il est du côté des livres de linguistique, quoique moins souvent sans doute que parmi ceux de la littérature romanesque, de ces grands fleuves tumultueux, qui, loin d'enserrer la réflexion du lecteur dans d'étroites limites, l'entraînent à plaisir, dans une grande bigarrure d'évocations et d'intuitions, bien au-delà de ses trop habituels rivages. Empathie et linguistique est de ces livres, et de ces débordements.

Le titre choisi par Robert Forest (R.F.) conjoint deux notions dont l'association est d'autant plus insolite qu'elle consiste en un pur accouplement paratactique, plutôt que de s'incarner dans le sage rapport d'inclusion qui eût conduit un auteur moins audacieux à opter pour L'Empathie en linguistique, voire pour L'Empathie dans la langue. À vrai dire, pareils titres auraient déjà été fort peu banals, car si fécond et novateur soit le programme de recherche conçu par Susumu Kuno il y a plus de vingt-cinq ans, la cohorte des linguistes enclins à convoquer explicitement et systématiquement la notion d'empathie, ne serait-ce que dans leur démarche heuristique, demeure, sinon clairsemée, du moins passablement dispersée. Outre Kuno et ses disciples, R.F. ne mentionne guère qu'une poignée de francs-tireurs, comme John Lyons et Anne ZribiHertz (p. 19); sans doute faudrait-il aussi évoquer un Claude Boisson (1987), qui 
propose au demeurant une analyse «empathologique» du verbe avoir à laquelle R.F. fait sans le savoir écho (p. 164). Qui plus est, l'empathie, en tant que mécanisme explicatif, n'a que sporadiquement étendu son terrain d'élection très au-delà de quelques topoi lexicaux assez bien circonscrits (par exemple les verbes de don en japonais, cf. p. 19) et d'un domaine syntaxique qui privilégie fortement pronominalisation et réflexivisation ainsi que passivation, transitivité et structuration de l'énoncé.

3 Le mérite le plus visible de Robert Forest est sans conteste de se jouer de ces bornages, non sans donner avec une réjouissante et impétueuse constance dans l'allégresse iconoclaste du défricheur et de l'explorateur au long cours.

4 S'il est d'emblée soucieux de ne point méconnaître sa dette intellectuelle envers Kuno, R. F. prend aussi grand soin (p. 17) de se démarquer du courant théorique dans lequel s'inscrivent des travaux aussi représentatifs que Kuno et Kaburaki (1977) ou Kuno (1987). À la démarche kunosienne, fondée sur une dissociation de la syntaxe d'avec l'énonciation, R.F. dit préférer une approche intégrative, davantage conforme assurément à la tradition française incarnée par Émile Benveniste ou Antoine Culioli. Mais son ambition est aussi, tout du long, ouvertement typologique: il s'agit de regrouper, sous l'égide de l'empathie, des phénomènes morpho-syntaxiques que la tradition traite sous des rubriques séparées, ne serait-ce que parce que chacun est d'ordinaire l'apanage des spécialistes de tel ou tel idiome ou groupe de langues. Il en va ainsi, tout particulièrement, du concept de version, que R.F. emprunte aux spécialistes des langues caucasiques et dont il se proposera, tout au long des cent dernières pages que remplissent les chapitres III à $\mathrm{V}$, de faire le principe explicatif de toute une série de dispositifs formels attestés dans des langues aussi diverses que le burushaski, le tagalog ou le hongrois.

5 Auparavant, après avoir rappelé dans l'Introduction les tribulations que le concept $d$ ' Einfühlung, ou d'empathie, a connues au fil des $19^{\mathrm{e}}$ et $20^{\mathrm{e}}$ siècles en psychologie et en esthétique, R. F. aura, dans le chapitre I, mis le concept d'empathie à l'épreuve de faits de langues qui paraissent taillés à sa mesure.

6 Ainsi, il est bien connu que les directionnels déictiques comme venir ou aller ont, d'une langue à une autre, vocation à situer un déplacement dans l'espace par rapport au site, sinon de l'énonciateur (appelé par R.F. «énonceur »), à tout le moins d'un participant avec lequel celui-ci «empathise » en quelque manière. L'auteur, ici comme à maintes autres reprises, pousse la réflexion très au-delà des commentaires, si pertinents soientils, auxquels donne habituellement lieu pareil sujet. On pense par exemple au rapprochement particulièrement suggestif qu'il opère entre la dichotomie ventif/itif et le couple adverbial schon/noch en allemand (p. 46-47). On en voudra aussi pour preuve l'application qu'il fait des concepts de «continuité » et de «rupture» du «fil empathique " afin de rendre compte du sémantisme des marqueurs, primitivement ventif et itif, de «co-agence » et de «disagence » en maricopa (p. 52-53); de fait, il s'agit là d'un cas exemplaire de grammaticalisation, ou plus exactement de «resémantisation »-cf. Ph. Bourdin (1992: 289-290).

7 Plus discutable, parce que trop allusif pour être convaincant, est le développement consacré p. 39 au turkana (f. nilo-saharienne). Il paraît un peu hâtif d'établir un lien entre la valeur «rétrospective» dont le marqueur itif est le support et un procès "obviatif», "qui fait irruption dans une sphère ». Sans doute R.F. pense-t-il ici au prétérit itif du catalan, dont il rend compte par ailleurs en termes d'« empathie 
contrariée» (p. 65-68), mais faute de démonstration empirique véritable, le rapprochement semble par trop audacieux, tout comme d'ailleurs l'emploi du qualificatif «obviatif», auquel les spécialistes des langues algonquiennes donnent une acception sensiblement plus restrictive. L'évocation du turkana s'inscrit dans une section très richement documentée portant sur les dérivations applicatives. Contre D. Creissels (1991), R.F. dénie à ces dernières le statut de diathèse, au motif qu'elles n'impliqueraient pas une véritable addition d'actant. Même si le point de vue de R.F. prête à discussion, l'amorce d'un tel débat est un indice, parmi bien d'autres qui vont se multipliant au fil du livre, de la réflexion approfondie que R.F. a consacrée à la catégorie de la diathèse. Au nombre de ces indices figure une analyse particulièrement éclairante du marqueur -(ra)re- en japonais, dont R.F. démontre que s'il est support du "passif», c'est en quelque sorte par accroc et à titre subsidiaire, suite à la contradiction, de nature "empathologique ", qu'il donne à voir entre la "centralité " du prime actant et son « absence de contrôle » (p. 41-43).

On serait mal venu de reprocher à R.F. l'insistance qu'il met à fonder ses hypothèses sur une prise en compte des marqueurs dans leur fonctionnement langagier effectif, loin des spéculations a priori et des taxinomies préalables. Pour ce qui est de la deixis directionnelle, cette observance de principe le conduit toutefois à une décision contestable, qui consiste à traiter séparément des marqueurs grammaticaux et des marqueurs lexicaux; ceci a pour effet de masquer l'unité profonde de la catégorie à laquelle ressortissent les uns comme les autres, unité dont témoignent éloquemment les filières de resémantisation dont ils participent. On pourra, à cet égard, se reporter à la défense et illustration du concept d'« hétérosémie » que propose Fr. Lichtenberk (1991 : 476) dans son étude sur le devenir historique des «équivalents » de 'venir' et 'aller' parmi les langues océaniennes :

«I will adopt (...) Persson's 1988 term 'heterosemy' to refer to cases (within a single language) where two or more meanings or functions that are historically related, in the sense of deriving from the same ultimate source, are borne by reflexes of the common source element that belong in different morphosyntactic categories (...). Thus for example, there is heterosemy if a verb, a directional particle, and an aspect marker all ultimately descend from the same historical source. "

['J'adopterai (...) le terme 'hétérosémie', emprunté à Persson (1988), pour désigner la prise en charge - dans une langue donnée - d'au moins deux significations ou fonctions historiquement apparentées parce que procédant d'une source primitive commune, lorsqu'elle est assurée par des avatars de la source primitive qui ressortissent à des catégories morphosyntaxiques distinctes (...). Par exemple, on parlera d'hétérosémie lorsqu'un verbe, une particule directionnelle et un marqueur aspectuel proviennent tous, en dernière instance, de la même source historique.']

Lichtenberk assortit cette définition d'une démonstration empirique serrée qui est de nature à invalider, dans une certaine mesure, le rigorisme méthodologique dont fait preuve R.F. sur ce point.

Quitte à être indûment sélectif, tant sont nombreux les êtres et processus langagiers scrutés ou simplement évoqués au fil de ce premier chapitre, on retiendra aussi le traitement des « explicateurs» du hindi en termes de «bilan empathique» (p. 69-77); dans le sillage d'A. Montaut (1991), R.F. va ici bien au delà des analyses aspectuelles classiques comme de celles qui s'en tiennent à ces effets de sens que sont la "précipitation " du prime actant, son absence de contrôle sur le procès, ou encore l'idée d'obstacle à surmonter. Le soin apporté à la sélection des exemples n'a d'égales que la force et la cohérence de l'argumentation. 
11 L'humeur picaresque de R.F. le conduit, dans le chapitre II, à arpenter tour à tour des terres aussi variées que les paradoxes interjectifs comme Dis donc! (p. 96), les hypallages du type un grand malade (p. 98-99), les emplois transitifs de tomber dans tomber la veste ou tomber les filles (p. 109), ou encore les contraintes d'îlot responsables $\mathrm{du}$ contraste d'acceptabilité entre des constructions allemandes comme das geheime Wahlrecht, "le droit de vote à bulletin secret », et *der vierstöckige Hausbesitzer, « le propriétaire d'immeuble à quatre étages » (p. 102-103). Périple ambitieux et lumineux tout à la fois, par l'originalité des perspectives qu'il ouvre, par l'ingéniosité du commentaire, par l'ardeur enfin mise à constamment pousser à la lisière extrême de ses ultimes retranchements la pertinence explicative du concept d'empathie et de ses dérivés : «transitivité empathique» et «solidarité empathique», sans oublier ces «tensions » ou « conflits empathiques » qui seraient à l'origine de certains emplois du cas ergatif dans les langues indo-aryennes (p. 118-119) comme de la substitution, fautive mais parfaitement motivée, de coupes sombres à coupes claires en français ordinaire (p. 120-121).

Deux réserves tout au plus, et d'inégale portée. Le développement consacré en fin de chapitre au ne explétif du français et à des tours connexes dans quelques autres langues souffre d'être quelque peu expéditif - à l'image de la toute dernière phrase, dont le rythme haletant n'est pas sans évoquer la course d'obstacles (p. 124-125). Plus gênant nous paraît être l'analyse qu'inspire à R.F. (p. 100) l'ambiguïté de l'énoncé suivant, en rapanui (f. austronésienne) :

\begin{tabular}{|l|l|l|l|}
\hline ko & aaNa & era & $? a$ \\
\hline perfectif & faire :RÉDUPL & il.y.a.longtemps & résultatif \\
\hline
\end{tabular}

«Cela a été fait intensivement autrefois.»

$\mathrm{Ou}:$ : Cela a été fait il y a très longtemps. »

13 Ayant précisé que la réduplication vocalique dont le verbe est l'objet engendre un effet d'intensification, R.F. note que cet effet peut porter soit sur le procès, soit sur l'éloignement temporel que dénote l'adverbe era. Le «transfert forcé de bilan empathique" qu'il invoque pour rendre compte de cette alternance de champ d'incidence implique, si nous comprenons bien, que le verbe et l'adverbe sont l'un et l'autre supports de point de vue, c'est-à-dire foyers empathiques. Outre que cela suppose une extension périlleuse - car intuitivement contestable - de ces notions, on peut se demander ce qui empêcherait dès lors d'invoquer le "transfert empathique » à chaque fois que, linéarité des formes oblige, il y a discordance entre l'attache formelle d'un marqueur donné et sa portée sémantique. Va-t-on ainsi, à propos d'un énoncé aussi banal que Jean était un homme courageux, parler de translation empathique (elle aussi grammaticalement imposée) au motif que l'imparfait est interprété soit comme signifiant que Jean a cessé de se montrer courageux, soit comme le reléguant dans la catégorie des chers disparus? On ne voit pas très bien ce que pourrait gagner à pareil débordement conceptuel une théorie en émergence de l'empathie langagière... dont R.F. a par ailleurs fort opportunément précisé qu'il n'entendait point la « mettre (...) à toutes les sauces » (p. 20).

Le chapitre III, consacré pour une large part à la « version » du verbe géorgien, tient un rôle charnière, en ce qu'il permet à R.F. d'introduire une figure de l'empathie dont il 
s'efforcera de démontrer, dans les deux chapitres suivants, le caractère en quelque sorte exemplaire, sinon prototypique. Se présentant comme des marques vocaliques précédant immédiatement le radical verbal, les versions ressortissent à l'empathie en ce qu'elles ont pour fonction d' " [orienter] les procès vers des points de vue d'entités virtuellement participantes» (p. 135), autrement dit de "mettre en perspective le procès (...) en l'orientant vers le "concernement" d'une entité » (p. 153). Ainsi, la substitution de la version $i$ à la version "neutre » $a$ dans un énoncé comme $v-a-d u V-e b$, "Je le fais cuire ", aura pour effet d'assigner à l'Agent une position de destinataire ou de bénéficiaire par rapport au procès : «Je le fais cuire pour moi » (p. 135); ce faisant, "[la version] "fait retour" sur la sphère d'un participant (...)» (p.136). Soucieux de montrer que les versions sont irréductibles à la catégorie de la diathèse, R.F. note que selon le profil morpho-syntaxique du verbe auquel il s'adjoint, le marqueur $i$ va alterner entre des valeurs moyenne, passive, et même antipassive : à v-a-gineb, "Je l'injurie ", s'oppose ainsi v-i-ginebi, 'Je profère des imprécations' (p.139).

15 N'étant pas «kartvélologue» (p. 151), nous ne saurions évaluer la pertinence de l'analyse que R.F. propose pour $i$ d'abord, puis pour les quatre autres versions. On se bornera à observer que les deux exemples cités plus haut montrent assez la propension des versions à déborder constamment tel ou tel des cadres taxinomiques dans lesquels on voudrait les enfermer, ce qui légitimise le scepticisme, au demeurant très constructif, que nourrit R.F. envers les traitements traditionnels. Et sa métaphore du "retour », ou de la boucle, nous semble apporter, au moins intuitivement, plus qu'un début d'explication à des faits tels que ceux évoqués par B. G. Hewitt (1995: 176) et déclarés par ce dernier « imprévisibles », à savoir par exemple l'alternance entre saxl-s $g a-q$ 'id-i, « Tu vendras la maison », et saxl-s $i-q$ 'id-i, 'Tu achèteras la maison'.

Lorsqu'on consulte une grammaire comme celle que J. Colarusso (1992) consacre au kabarde, on comprend pourquoi R.F. refuse (p. 155), en termes trop sibyllins pour ne pas piquer l'attention du lecteur, de souscrire à l'exploitation qui a été faite du concept de version par les spécialistes des langues caucasiques du nord-ouest. Ce qui est présenté par J. Colarusso (ibid., p. 96-97) comme "version» du kabarde n'est autre qu'une sorte de postposition interne au complexe préverbal $\left(-\chi^{\wedge} \mathrm{w} a-\right)$, qui alterne entre valeur bénéfactive et détrimentielle. Le paradoxe - au moins terminologique - est que ce marqueur peut fort bien coexister avec le préverbe -q"-, étiqueté "horizon of interest » par J. Colarusso et dont le fonctionnement sémantique évoque la version géorgienne bien plus nettement que ne fait $-x^{\wedge} a a-$ : ainsi, l'insertion de $-q^{\prime \prime}-$ dans un énoncé traduit " Je t'ai donné le livre » appellera la glose «Je t’ai donné le livre, mais il demeure dans mon domaine d'intérêt» - autrement dit, "Je t'ai prêté le livre » (cf. J. Colarusso, ibid., p. 85). On comprendra que le marqueur kabarde $-q$ "- nous paraisse être de ceux qui gagneraient sans doute à être versés (sans jeu de mot) au compte de l'empathie.

17 Faute d'espace, on passera sur l'analyse empathologique des préfixes verbaux du burushaski, qui clôt le chapitre III et étend à cette langue la logique versionnelle dégagée pour le géorgien, avant d'être appliquée au lahu (f. sino-tibétaine) et au sanuma (l. du Brésil apparentée au yanomami). Ici encore, le raisonnement de R.F. nous a paru marier subtilité et rigueur.

18 Dans le chapitre IV, R.F. se livre d'abord à une critique finement argumentée de la terminologie en usage chez les spécialistes du tagalog, et notamment de l'utilisation qu'ils font des notions de «voix» et de «focus ». Le propos débouche rapidement sur 
une réflexion bien plus générale, qu'inspirent à R.F. le concept syntaxique de sujet et la prétendue universalité de ce dernier. On retiendra une ou deux formules qui résument avec bonheur sa pensée : ainsi (p. 172), lorsqu'elle est opératoire dans une langue, « la fonction sujet a pour effet de constituer une sorte de pôle extraprédicatif dans l'énoncé " (souligné par nous, Ph.B.) ; ou encore (p. 173) : «Le sujet se définit toujours comme l' actualisateur principal. Vient-on à manquer de critères pour poser ce caractère principal, alors on sera bien avisé de renoncer carrément à parler de sujet » (souligné par nous, Ph.B.). C'est très précisément à cette renonciation qu'invite, selon R.F., une langue comme le tagalog - et en cela il se sépare nettement d'A. Lemaréchal (1989). L'argument prétendument "focalisé» ne serait rien d'autre qu'un «centre empathique », et la marque de "voix» dont le verbe est porteur une version, dont la fonction est d' «anticiper» ce centre (p. 175) et d'expliciter son statut actanciel, autrement dit « en tant que quoi (...) le référent du centre empathique est concerné (...) par le procès » (p. 174). Les faits sur lesquels R.F. assoit son argumentation (p. 176-179) sont à l'intersection de la syntaxe et de la sémantique, le recours aux concepts de version et de centre empathique permettant de rendre raison du comportement, autrement énigmatique, de certains verbes qui ne présentent pas la même marque de "voix", ou plus exactement de version, selon qu'ils apparaissent en proposition indépendante ou dans une relative. À cet argument intra-langagier s'en ajoutent d'autres, que livre l'investigation typologique menée dans les dernières sections du chapitre; on retiendra, pour leur caractère conceptuellement stimulant, les développements consacrés au malgache, au tsimshian (f. penutia) et à la construction participiale de l'italien (p. 180-190).

19 Si l'auteur prend soin de distinguer les concepts de sujet et de centre empathique, il leur reconnaît une caractéristique commune, à savoir leur unicité (p. 172). La question est d'importance, car dans le chapitre d'Introduction R.F. a brièvement exposé les raisons du scepticisme que lui inspire l'idée, kunosienne, selon laquelle il y aurait « par énoncé un seul authentique foyer d'empathisation » (p. 26). Il nous semble qu'il y a là à tout le moins, et à quelque 150 pages de distance, comme un flottement. Sans doute l'hésitation de R.F. est-elle un corollaire de l'extension très large qu'il assigne au concept d'empathie et qui est elle-même une conséquence du caractère exploratoire de sa démarche. Reste que cette question de l'unicité demeure non résolue - pour autant qu'elle le soit, au demeurant, chez les kunosiens : cf. S. Makino (1976).

La réflexion sur la « voix » moyenne, amorcée dans le Chapitre III à propos du géorgien, trouve son point d'orgue dans le Chapitre V. La thèse essentielle de R.F. est que le moyen ne ressortit pas à la voix, mais qu'il est une version, et jusqu'à un certain point «la version des langues sans versions»(p. 194). Entre autres arguments formels, l'auteur fait état d'une dissymétrie entre passif et moyen qui est de fait remarquable: tandis que les langues confient volontiers le marquage du premier à un auxiliant, très rares sont celles qui usent de ce procédé pour le moyen (p. 195-196). Moins convaincant peut-être est le développement consacré au peul, langue nigéro-congolaise où la tripartition actif/passif/moyen est entérinée par un système de suffixes verbaux qui s'impose avec trop d'évidence pour que les lézardes invoquées par R.F. suffisent à remettre en question, comme il nous y invite, l'édifice tripartite lui-même (p. 199-201). Suit une série de remarques sur les avatars du moyen dans les langues indoeuropéennes, et notamment sur la coïncidence formelle entre moyen et réfléchi dans plusieurs d'entre elles, dont le français et l'espagnol (p. 207-208). Il y aurait quelque injustice à « épingler » telle ou telle observation, dans la mesure où R.F. ici inventorie 
plutôt qu'il n'argumente. Ainsi, il est possible que la réanalyse du moyen comme une version empathique permette, comme il le suggère, de rendre raison des effets sporadiquement contrastés du marqueur réfléchi en français (intentionnalité de se plier (à)) et en castillan (inintentionnalité de caerse), mais l'approche de R.F. est ici trop allusive, sinon "papillonnante ", pour déboucher sur des propositions réfutables. Du moins a-t-elle le mérite, qui n'est pas mineur, de dessiner les contours d'une problématisation neuve et potentiellement féconde. Telle est aussi l'ambition de l'hypothèse sur l'«anti-moyen" en hongrois, qui donne lieu, quant à elle, à une argumentation serrée et audacieuse (p. 210-220), alors même qu'elle témoigne, par le choix même du terme "anti-moyen", de la difficulté qu'éprouve R.F. à se détacher complètement du cadre mental que nous impose la taxinomie traditionnelle, dans son insistance, par ailleurs dénoncée, à ranger le moyen parmi les « voix ».

Grand fleuve bigarré, disions-nous plus haut, que nourrit la luxuriance du style - on pense, entre maints exemples, à ce "pseudopode empathique» que le procès "s'adjoint » à lui-même (p. 36) - et qu'anime la résolution, roborative et sans cesse réaffirmée, d'embrasser la complexité des faits de langues, y compris dans ce qu'ils ont d'irréductible aux généralisations orthodoxes et commodes. Sans oublier non plus, il est vrai, les quelques débordements polémiques auxquels il advient à R.F. de céder çà et là, non sans trivialité parfois (p. 56), ni même, à l'occasion, sans outrance dans l'imprécation (p. 147). Ce sont là des excès qui au vrai paraîtront subalternes, comme l'est la seule erreur que nous ayons pu relever parmi les très nombreux faits de langues invoqués par l'auteur tout au long de ces 220 pages (et dont le présent exposé n'a évoqué qu'un minime échantillon): loin d'être irrecevable (cf. p. 197), un énoncé comme He drowned est en anglais, tant nord-américain que britannique, le moyen le plus banalement grammatical de décrire une mort par noyade. Tout aussi vénielles sont les quelques lacunes relevées dans la bibliographie: nous voulons parler du mutisme qu'elle observe à propos de cinq auteurs référencés dans le corps du texte, à savoir A. Abeillé 1996 (p. 112), N. Marr 1908 (p. 136), Ch. Li 1976 ( p. 167), M. Arce-Arenales 1989 (p. 208), et J. Perrot 1993 (p. 218).

Failles mineures que celles-là, dans un livre incontestablement majeur.

\section{BIBLIOGRAPHIE}

Ne sont recensés que les textes cités ci-dessus et non mentionnés dans le livre de R.F.

BoIsson, Cl. (1987), « Anglais « have », français « avoir » et l'empathie », in La Transitivité (Domaine anglais), Université de St-Étienne, Travaux du CIEREC (Vol. 52), p. 155-180.

BOURDIN, Ph. (1992), «Constance et inconstances de la déicticité : la resémantisation des marqueurs andatifs et ventifs ", in M.-A. Morel et L. Danon-Boileau, s. la dir. de, La Deixis, Paris, P.U.F., p. 287-296.

COLARUSSO, J. (1992), A Grammar of the Kabardian Language, Calgary (Alberta), The University of Calgary Press. 
HEWITT, B. G. (1995), Georgian: A Structural Reference Grammar, Amsterdam et Philadelphie, John Benjamins.

LICHTENBERK, Fr. (1991), « Semantic change and heterosemy in grammaticalization », Language, 67: 3, p. 475-509.

MAKINO, S. (1976), « Can a single sentence have more than one empathy focus? - A case study from Japanese », Papers from the Twelfth Regional Meeting, Chicago Linguistic Society, p. 476-485.

\section{AUTEURS}

PHILIPPE BOURDIN

Université Paris X - Nanterre

et Université York, Toronto 